\title{
Zum Umfeld von August Sauer: der Germanist Joseph Körner ${ }^{1}$
}

Nicht als verdienter Romantik-Forscher, Epistologe, Herausgeber, Kämpfer auf methodologischen Feldern, nicht als Kontrahent August Sauers, Josef Nadlers und Herbert Cysarz', nicht als Korrespondenzpartner Schnitzlers und Kafkas, nicht als Zeitzeuge und Opfer antisemitischer Säuberungsaktionen der Nazis steckt mir Josef Körner im Bewusstsein seit 1999 (also noch aus der Zeit vor der verdienstvollen Herausgabe von Josef Körners Pbilologischen Schriften und Briefen durch Ralf Klausnitzer) (Körner 2001), sondern als Fall bedauernswürdiger Schlampigkeit unsererseits (eine Straftat im Philologischen, die gerade Körner aufs strengste verfolgte und in seinen Rezensionen schonungslos bemängelte): Als wir in der Olmützer Arbeitsstelle für deutschmährische Literatur den ersten Band des Lexikons deutschmährischer Autoren (Fiala-Fürst u. a. 2002) herausgaben, passierte es, dass wir den Aufsatz zu Körner, den uns aus Saarbrücken Gerhard Sauder schickte, einem anderen Autor zuschrieben. Die Betroffenheit war groß, die Anstrengungen, es wieder gutzumachen, blieben erfolglos.

Zum zweiten Mal dämmerte mir der Name Körners auf, als ich 2003 in der Funktion des Prodekans für Wissenschaft und Forschung in unserer Fakultät die schandhafte Habilitations-Kausa des Troppauer Bohemisten Pavera zu verwalten hatte. Obwohl sich die beiden - in ihrer Zeit jeweils Furore machenden - Habilitationsfälle freilich nicht vergleichen lassen (denn Pavera reichte ein Plagiat ein, das wohlwollend - weil unwissend - aufgenommen wurde, während Körner ein Originalwerk vorlegte, das böswillig abgelehnt wurde - dazu später), nahm hier die „Vermährischung“ Körners (die wir in der Olmützer Arbeitsstelle lustvoll mit vielen Autoren betreiben) ihren Lauf. Denn obwohl Körner die meiste Zeit seines Lebens in Prag verbrachte, nur wenig „Mährisches“ schrieb und sicher nicht für den „mährischen Sauer“

1 Der Aufsatz ist im Zusammenhang des Projektes Výzkumný zámèr Morava a svět. Umèní v otevreném multikulturnim prostoru [Mähren und die weltliche Kunst im offenen multikulturellen Raum], MSM 6198959225 entstanden. 
erklärt werden kann, ist er mir schicksalhaft zum „mährischen, zum Olmützer Fall“ geworden. ${ }^{2}$

Alles, was einen Sauer-, Nadler-, Kafka-, Schnitzler-Forscher, einen Romantik-, einen Methodologie- und Germanistik-Historiker, einen Kenner der Prager deutschen Literatur an Körner interessieren könnte, ist bereits - in früherer und neuester Zeit - von Eisner, Fischer, Wellek, Klausnitzer, Eichner, Krolop, Fliegl, Härtl, Sauder, Fohrmann gesagt worden. Freilich nicht in zusammenfassender Art etwa eines würdigenden Sammelbandes: Eine selbständige Körner-Tagung und ein Körner-Sammelband würden sich lohnen, denn auf einem solch zeitlich wie räumlich breiter abgesteckten Feld könnte man die (in den genannten Schriften manchmal nur angedeuteten) Thesen und Themen des Körnerschen Werkes breiter ausführen, die methodologischen und weltanschaulichen Kämpfe innerhalb der germanistischen Kreise seit Scherer beleuchten, die Positionen der Prager germanistischen Ordinarien innerhalb dieser Kreise absteckten, das komplizierte Knäuel der deutsch-tschechisch-jüdischen Beziehungen im Prag der Zwischenkriegszeit ansatzweise entwirren. Außerdem verdient Josef Körner, ein großer Kenner der deutschen Romantik, glücklicher Entdecker und verlässlicher Herausgeber verschollen geglaubter romantischer Texte, bissiger, brillianter Rezensent, schonungsloser Kritiker aller Verstöße seiner germanistischen Zeitgenossen gegen wissenschaftliche Sauberkeit, fleißiger Begleiter neuester Literatur und Literaturwissenschaft, Josef Körner, ein bezugsreicher Mann, der am Ende sein Leben und Werk trotzdem nicht anders bewerten konnte als einen Scherbenhaufen, verdient unsere Aufmerksamkeit und Erinnerung.

2 Als ich noch anhand der Lebensdaten (Körner, Jahrgang 1888, besuchte das deutsche Staatsgymnasium in Ungarisch Hradisch von 1898 bis 1906), schloss, dass mein jüdischer Großvater (Jahrgang 1890) Körner wohl aus der Schule kannte, denn auch er besuchte das deutsche Staatsgymnasium in Ungarisch Hradisch, war mir endgültig klar, dass Körner auf geheimnisvoll-mystische Weise mit mir zusammenhängt. 


\section{Romantik}

Kurt Krolop nennt die Romantikforschungen Körners an erster Stelle in seiner Rezension zu Klausnitzers Herausgabe von Körners Schriften, gleichsam als das einzig Übriggebliebene:

Josef Körner, ein Germanist, dessen Name über einen engeren Kreis von kundigen Romantikforschern hinaus wohl [nicht mehr] allgemein geläufig sein dürfte. (Krolop 2004: 265)

Trotz der großen Menge der Romantikaufsätze Körners, trotz der geglückten, in seiner Zeit spektakulären Manuskriptfunde ${ }^{3}$ und trotz deren akribischer und verlässlicher Edition, auf die freilich die spätere Romantikforschung mit Gewinn zurückgriff, wage ich zu behaupten, dass gerade die Romantikforschungen Körners das am wenigsten Bleibende, am wenigsten Frische und Lebendige in seinem Gesamtwerk ist: Seine Einzelinterpretationen (etwa die als zweite Habilitation eingereichte zu Kleists Michael Koblhaas und Prinz Friedrich von Homburg) wurden mit der Zeit durch luzidere, spannendere, modernere überboten, Körners „versuchte Ehrenrettung der romantischen Denker (v. a. der Schlegels) galt einer anderen Epoche und geistigen Lage, als es die heutige ist (was bereits im Jahre 1938 Max Horkheimer [1938: 220] sah, der sie in seiner kurzen Besprechung der Körnerschen Schrift Krisenjahre der Frübromantik [1937] als „sehr post festum" glossierte) und ist eher an den - eindeutig lesbareren oder eindeutig witzigeren - Darstellungen einer Ricarda Huch oder eines Egon Friedell zu messen als am Stand der heutigen Romantikforschung.

Andererseits stimmt es aber, dass Körners lebenslange Hingabe an die romantische Epoche sein ganzes Werk lenkte und sogar - wie ich später zu beweisen versuche - sein Germanistenschicksal maßgeblich beeinflusste.

3 Wie Klausnitzer darlegt, machte Körner besonders der „Coppeter-Fund“ berühmt: Im Sommer 1929 entdeckte er im Schloss Coppet am Genfer See an die 3500 Briefe August Wilhelm Schlegels aus der Zeit 1804-1812, doch bereits als frischer Promovent fand er in den Bibliotheken in Wien, Dresden und Trier große Teile des Nachlasses Friedrich Schlegels und rief die - zwar kurzlebige, doch wichtige - Wiener romantische Zeitschrift Friedensblätter ins Gedächtnis der germanistischen Nachwelt usw. 


\section{Noch einmal Romantik: Expressionismus und Prager deutsche Literatur}

Das überhaupt spannendste am Thema Romantik ist, wie Körner die Romantik mit den zeitgenössischen literarischen Epochen, sprich dem Expressionismus, verbindet. Freilich ist Körner nicht die Urheberschaft an dieser gedanklichen Verbindung (Romantik-Expressionismus) zuzuschreiben, denn sie ist zeitund epochensignifikant, und freilich ist mir nicht das Primat zuzuschreiben, ich wäre die erste, die bemerkte, dass Körner expressionistische Werke, romantisch' interpretiert. Ralf Klausnitzer (2001: 411) macht auf diese Tatsache bereits aufmerksam, indem er auf die „Parallele zwischen romantischen und expressionistisch-gegenwärtigen Heilserwartungen“ hinweist, die Körner im Aufsatz über Zacharias Werner ziehe. Doch die Parallelisierung geht weiter, ist tiefer, verdient eingehendere Betrachtung:

Körner lobt, bemerkt an expressionistischen Werken, die er fleißig las und rezensierte, ${ }^{4}$ vor allem (überhaupt nur) diejenigen Eigenschaften, die ihn an romantische Ästhetik und Programmatik erinnern. Den ethischen Ernst:

Das ist anders geworden, seit allerjüngste Literatenkreise Dichtung wieder so ansehen und werten, wie einst Friedrich Schlegel, Novalis, Schelling. Daß die Wortkunst der Ort für das Religiöse und Ethische und alle geistige Erkenntnis [...] sei-die gleiche strenge Forderung spricht aus dem Programm des Athenäum wie aus dem Bekenntnisse derer um die Weißen Blätter, die Aktion, die Neue Jugend (Körner 1918: 359),

die religiöse Zielsetzung:

Beiden ist es letzten Endes weniger um künstlerische Gestaltung ihrer Empfindungen zu tun, als um religiöse Erschütterung und Erneuerung der Menschheit, von christlicher Sendung [5] träumen und reden sie, (Körner 1923: 52) ${ }^{6}$

den a-mimetischen Mut: „Das junge Geschlecht unserer neuesten Poeten kümmert sich wenig um getreue Abschilderung irgendwelcher Wirklichkeiten“

4 Georg Kaiser, René Schickele, Paul Adler, Franz Werfel, Paul Kornfeld, Max Brod, Franz Kafka, Arthur Schnitzler u. a.

5 Dies ist freilich ein Hinweis auf Werfels Aufsatz von 1918.

6 Zugleich tut Körner noch eine Parallele auf, indem er auf die Gefahr - damals wie heute der Übersättigung der Dichtung mit geistig-religiösen Inhalten hinweist: „Aber die Gefahr, der vor Zeiten die Frühromantik erlag, beginnt auch der neuen Generation gefährlich zu werden: Daß Übermaß des Geistigen das Gefühlsmäßige, der Prophet den Dichter verdrängt.“" (Körner 1918: 359) 
(Körner 1918: 762), das Primat der Phantasie, „Spontaneität der Seele [...], „Empfindungsintensität,“ (Körner 1917a: 776f.) Befreiung des Gefühls, Pathos, Sentimentalität, und weitere ähnliche Stützpunkte mehr.

Diese Erkenntnis, Körner misst den zeitgenössischen Expressionismus an der Romantik, ist auch die Erklärung dafür, wieso Körner - trotz der fleißigen, einfühlsamen Lektüre expressionistischer Werke - kein Verständnis für expressionistischen Stil und expressionistische Sprache hatte. „Die verletzende Ungebundenheit der Problematik" in Kaisers Versuchung lässt er gerade noch durchgehen, weil ihn der Aufbau dieses Dramas an Lenz' Soldaten erinnert, doch ansonsten sind ihm Kaisers Dialoge „halbdunkel“ und „ungeschickt" auf einzelne Figuren verteilt, Gestalten ,nicht klar hervortretend“ und „,banal“ (Körner 1917b: 762). Werfels lyrische Sprache wiederum - in den ansonsten ekstatisch gelobten ersten drei Werfelschen Sammlungen - bezeichnet er als „kunstlos und karg“, seine Bildschöpfungen als ärmlich, Werfel gefalle sich „ohne ersichtlichen Grund in skurrilen Wortfügungen, die schon an der letzten Grenze der Begreifbarkeit, sowohl als der Sprachmöglichkeit stehen.“ (Körner 1917a: 782f.) Immerhin entdeckte Körner den Mechanismus und die eigenartige Wirkung des expressionistischen Reihungsstils (ohne freilich diesen viel jüngeren Terminus zu benutzen), ${ }^{7}$ alles in allem aber stand er der expressionistischen Erneuerung der Sprache sowie der Form ziemlich verständnislos gegenüber.

Und noch ein Merkmal der romantischen Epoche stellt Körner als Prisma vor seinen Blick auf die allerjüngste Literatur: die Gruppenbildungen. Bereits das oben angeführte Zitat über die ähnliche Anlage der vom Athenäum und der von der Aktion deutet diese Sichtweise an. Noch deutlicher ist diese Präfabrikation in der Charakteristik der Prager deutschen Literatur des expressionistischen Jahrzehnts zu sehen:

Wirkten Adler, Salus, Rilke, Meyrink jeder einzeln für sich und von sich aus, so sind Oskar Baum, Max Brod, Rudolf Fuchs, Franz Kafka, Otto Pick, Franz Werfel durch jahrelange, zum Teil bis in die Kindertage zurückreichende Freundschaft miteinander verbunden, denken und dichten gemeinschaftlich, beurteilen und fördern einander in treuer Kameradschaft. (Körner 1917c: 63)

7 „Das feste Rückgrat von Werfels Sprache bildet das markige, stets in Massen aufgebotene Substantivum [...] an die Gruppengestalten dieses Malers [Egger-Lienz; IFF] erinnert Werfel umso mehr, als er nicht nur das einzelne Wort, sondern auch die Bilder [...] immer in ganzen Reihen vorführt, nicht darauf bedacht, jedes abgesondert auszupinseln, sondern gerade durch die im einzelnen ungestalte, als Ganzes freilich wohlkomponierte Masse zu wirken. Diese Technik verleiht der Werfelschen Lyrik den eigenartigen Litanaienton.“ (Körner 1917a: 784). Den Terminus „Reihungsstil“ führte m. W. in die Expressionismusforschung erst in den 60er Jahren Walter H. Sokel ein. 
Diese kollektivisierende Sicht auf die letzte Generation der Prager deutschen Literatur hat in den nachfolgenden Jahrzehnten Karriere gemacht, ist tausendmal repetiert worden und wird bis heute oft bemüht, obwohl inzwischen eingehende Forschungen belegten, dass bei weitem nicht alle Prager deutschen Autoren innerhalb der Freundeskreise beheimatet waren, sondern viele und vieles viel eher außerhalb und abseits stand. Spannend ist, dass gerade derjenige, welcher 50 Jahre später seinen Rückblick im genannten Sinne kollektivisierend auffasste und nannte, der Autor der literarischen Memoiren Der Prager Kreis, Max Brod, gegen diese Etablierung des Prager Kreises durch Josef Körner am heftigsten protestierte:

Ein Zettelsammelnder Literaturhistoriker, Professor Körner hat [...] dieses verzerrte Bild von mir in Umlauf zu bringen begonnen [...] Die Wahrheit ist, daß die meisten von den Autoren, die ein Mißbrauch mit mir in Zusammenhang zu bringen pflegt, weder meine Schüler, noch meine Freunde, nicht einmal Wohlwollende sind.“ (Brod 1918/19, zit. n. Klausnitzer 2001: 401f.)

Klausnitzer sieht die Heftigkeit der Reaktion Brods im Kontext der „kulminierenden Auseinandersetzungen mit Franz Werfel“" (Klausnitzer 2001: 402), was sicher auch richtig ist, doch meine Erklärung ist noch eine andere: Brod hat Körners Motivation zum Ausrufen, zum Installieren des „Prager Kreises“ aufgrund der Parallelisierung mit dem Romantikerkreis weder gesehen noch begriffen. Wäre ihm aufgefallen, dass Sätze wie

Max Brod ist vielleicht nicht der begabteste, gewiss aber der vielseitigste unter den Genossen [und] außerordentlich energiebegabt, ein Organisationstalent sondergleichen, ist er zum Führer der Freunde prädestiniert. Er ist zugleich die interessanteste und problematischste Figur seines Kreises (Körner 1917c: 63),

ihn, Max Brod, zum August Wilhelm Schlegel, Hugo Bergmann zum Schelling oder gar Fichte, Werfel zum Tieck und Kafka zum Novalis des Prager Kreises machten, hätte er sich vielleicht nicht so sehr aufgeregt. ${ }^{8}$

Zugleich zuerkennt Brod aber Körner - freilich in dementierender Absicht die Urheberschaft an der Erfindung des Prager Kreises: „Ein Zettelsammelnder Literaturhistoriker, Professor Körner hat [...] dieses verzerrte Bild von mir in Umlauf zu bringen begonnen " - Nachfolger und Nachplapperer dieser Körnerschen Gruppenthese folgten in Scharen nach bis auf die heutigen Tage, ohne meistens von Körners Urheberschaft das Mindeste zu wissen.

8 Körner hat übrigens im Jahr 1924 ,zurückgebissen“, als er im Aufsatz Erlebniß - Motiv - Stoff die Werke Max Brods, ,eines rein denkerhaft veranlagten Schriftstellers“, „, totgeborene Kinder" nennt - eine Metapher übrigens, die er sechs Jahre früher ähnlich für die Charakterisierung der Zauberflöte Paul Adlers benutzte (Körner 1918: 359). 
Und - kann man nicht genauso gut Körners einleitende Sätze in seiner Prag-Studie,

Einzigartig unter allen Städten Europas ist Böhmens schöne Metropole. Zweifaches, oder noch besser: dreifältig verschiedenes Leben spielt sich ab in ihren Gassen und Gebäuden. Zwei, ja drei nach Wesen, Ziel und Geschichte getrennte Kulturkreise schließt das eine Weichbild in sich ein (Körner 1917c: 55)

als die erste Postulierung der - genauso umstrittenen wie Schule machenden -,,Theorie des dreifachen Ghettos" ansehen, obwohl freilich das eigentliche Wort „Ghetto“ noch nicht fällt? Ein Jahrzehnt vor Paul Eisner?

3. Romantik zum dritten: Romantiker versus Klassiker. Die Habilitationsaffäre

Den Hergang der Habilitationskausa beschreibt Ralf Klausnitzer eingehend und akribisch. Hier also nur eine kurze Zusammenfassung: 1924 legte Josef Körner der Habilitationskommission unter der Führung August Sauers seine Habilitationsschrift Romantiker und Klassiker vor, die Sauer jedoch zerpflückte, in seinem Publikationsorgan Euphorion vernichtend rezensierte (Sauer 1925) und sie als Habilschrift ablehnte. Körner berief sich auf das Schulministerium (in seiner Eingabe führt er als Grund zur Ablehnung persönliche Animositäten auf - nicht genuine wissenschaftliche Urteile). Körner unterstützend meldeten sich namhafte Germanisten aus dem In- und Ausland zu Wort; Aufsätze, welche Körners Arbeit lobten, provozierten Aufsätze, die den Standpunkt der Prager Professoren verteidigten. Aus der Kausa entwickelte sich eine regelrechte germanistische Affäre, die den Ruf der Prager Germanistik zu beschädigen drohte. Sauer wurde vom Dekan der Philosophischen Fakultät zur Eröffnung eines Rekursverfahrens gezwungen: Allerdings wurde die Arbeit Körners erneut als „dem Standpunkte unserer gegenwärtigen Wissenschaft bezüglich der deutschen Romantik in keiner Weise entsprechend“ und ,als nicht geeignet,

9 Mitglieder der Kommission waren neben Sauer Adolf Hauffen, Erich Gierach, Gerhard Gesemann und Josef Wihan. 
das Ansehen der deutschen Literaturgeschichte zu fördern“, ${ }^{10}$ abgelehnt. ${ }^{11}$ Nach Sauers Tod (1926) reichte Körner 1927 - nachdem er sich gezwungenermaßen für seine vormaligen Proteste bei der Leitung der Universität entschuldigt und eine Deklaration über seine deutsche Nationalität abgegeben hatte (Klausnitzer 2001: 442) - eine andere Schrift ein, die erwähnte Kleistinterpretation, die schließlich von der neuen Kommission unter der Leitung Herbert Cysarz', des Nachfolgers Sauers im Amt, angenommen wurde. Cysarz - Körner nicht viel wohlwollender gesinnt als Sauer ${ }^{12}-$ ließ den Habilitanden aber bei der Prüfung durchfallen, was (damals wie heute) ein höchst ungewöhnlicher Vorfall war. Erst nach Protesten einiger Prager Ordinarien durfte Körner die Prüfung wiederholen und erreichte 1930 endlich die „venia docendi“.

Heute, nach 80 Jahren, müsste Körners Fall eigentlich nur als eine von vielen bedauernswerten akademischen Schändlichkeiten (wie wir sie bis heute erleben) eingestuft werden, doch er stachelt offensichtlich die germanistische Nachwelt nach wie vor zu Spekulationen ob der Gründe der Sauerschen Ablehnung an, wobei einzelne Deuter jeweils die eine oder andere Ursache für die wichtigste halten:

So hielt Körner selbst für die eigentliche Ursache des Sauerschen Missmutes seine (Körners) heftige Kritik an Sauers Lieblingsschüler Josef Nadler, dessen Literaturgeschicbte der deutschen Stämme und Landschaften er 1919 vernichtend rezensierte. Im Lichte der späteren steilen und bedenklichen Karriere der Nadlerschen Literaturgeschichte ist Körner seine Kompromisslosigkeit hoch anzurechnen, mit welcher er Nadler (gelinde formuliert) wissenschaftliche Unsauberkeit vorwirft, „die üble Manier [...] aus zweiter Hand geholtes Material zu verallgemeinern,“ „im Haschen nach schmetterlingsbunten Ideen den festen Boden der Tatsachen unter den Füßen zu verlieren“, , willkürlich und vorschnell“ zu urteilen, die Wahrheit der Dogmatik zu opfern, „dem Systemzwang zuliebe [...] hier Widerstrebendes [zu] harmonisieren und [zu] verbinden, dort Gleichförmiges gewaltsam auseinander[zu]zerren,“ Effekthascherei, welche das „leidenschaftslose Nachforschen der Wahrheit" durch „prunkvolle Inszenierung, in bengalische Lichter getaucht, von schwüler Rhetorik musikalisch akkompaniert" ersetzt. „Mit Wissenschaft hat sol-

10 Aus dem Gutachten des Frankfurter Ordinarius Franz Schultz (zit. n. Klausnitzer 2001: 432f.).

11 Körners Schrift erschien noch 1971 in der Reedition der WBG, was von deren Modernität und zugleich von der Verblendung und wohl tatsächlicher persönlicher Abneigung der damaligen Gutachter zeugt.

12 Wie man aus Cysarz' (1957) Memoiren eindeutig heraushören kann. 
ches Verfahren kaum noch etwas gemein“, urteilt Körner (1919) unbeirrt und erntet fünf Jahre später die Früchte seiner kritischen Lust.

Manche Interpreten halten die jüdische Herkunft Körners für den Grund der Querelen und schreiben hiermit Sauer eine antisemitische Motivation zu. ${ }^{13}$ Es mag zwar stimmen, dass Sauer kein großer Freund der Juden war, doch zu öffentlichen antisemitischen Äußerungen ließ er sich (die Kenner des Sauerschen Werkes sind gefragt) wohl doch nie hinreißen. Mit Krolop (2004: 277 u. 276) bin ich der Meinung, ${ }^{14}$ dass andere Gründe als eine ausgesprochene, antisemitisch kodierte Abneigung gegen den ethnischen - gar nicht orthodoxen - Juden Körner Sauers Urteil leiteten. Zu gerne würde ich Sauer die Adolf Bartelssche Einstellung nachsagen,

Ein Jude kann in der Tat nur eine Kritik, keine Geschichte der deutschen Literatur liefern, denn er weiß ja nicht, was uns notwendig war und ist. (zit. nach Fohrmann 1989: 237)

Doch dies wäre zugleich ahistorisch wie wahrscheinlich unrichtig, der Einstellung Sauers zum Judentum seiner Kollegen doch nicht entsprechend.

Was Sauer an Körner viel mehr hat stören müssen als seine jüdische Herkunft war Körners „unzuverlässige“, wenn nicht gar „verräterische“ Haltung im „Kampf ums deutsche Prag“ (die aber Sauer m. E. eben der jüdischen Herkunft Körners zuschrieb). Als Sauer Julius Petersen um ein Gutachten für das Rekursverfahren bat (welches Petersen allerdings ablehnte zu schreiben), legte er ihm nahe, er solle „den nationalen Gesichtspunkt in den Vordergrund stellen,“ denn bei Körner handele es sich um ein „,̈̈belbeleumdetes anationales Individuum", wohl mit Sympathien für Sozialisten und Zionisten (Boden 1994: 91).

Josef Körner schrieb, handelte und gab sich tatsächlich ziemlich anders, als man von einem deutschen Germanisten in der „bedrohten Festung des Deutschtums" erwartete, in der Prager deutschen Universität, deren deklariertes Ziel es spätestens seit der Trennung 1882 war, den Besitzstand der deutschen Kultur und Sprache gegen „Tschechisierungstendenzen“ zu stärken und zu verteidigen - eine Aufgabe, die gerade August Sauer mit aller Kraft erfüllte. Josef Körner kümmerte sich jedoch wenig um vorgezeichnete

13 Etwa René Wellek und Konstanze Fliedl.

14 Krolop legt sogar dar, dass Sauer bei radikalen völkischen Studenten „wo nicht geradezu als Jude, so doch als eindeutig philosemitisch galt"; zugleich erinnert Krolop an die Tatsache, dass zur gleichen Zeit, als Sauer Körner ablehnte, er einen anderen Germanisten jüdischer Herkunft problemlos habilitieren ließ - seinen Schüler Georg Stefansky. Diese Koinzidenz erklärt René Wellek wiederum als „Professor Bernhardis-Problem“: „under the circumstances of the time he could not possibly have two Jewish Privatdozenten." (zit. n. Klausnitzer 2001: 425) 
Linien und eingeschärfte Doktrinen, sondern pflegte unbekümmert freundschaftlichen Verkehr mit tschechischen Germanisten, las und rezensierte fleißig deren Schriften (v. a. Otokar Fischers) und wurde reziprok von ihnen wahrgenommen (die gesamte tschechische Germanistik, Josef Janko, Vojtěch Jirát, Otokar Fischer, stellte sich solidarisch hinter Körner in der Habilitationsaffäre), ${ }^{15}$ ließ in seinen Studien nie antitschechische Töne erklingen, sondern lobte im Gegenteil alle literarischen Bemühungen, die beiden Kulturen aneinander näher zu bringen (so z. B. die Texte Franz Werfels oder Jakob Julis Davids) - nahm somit eigentlich die archaische Bolzanosche Position ein und setzte sich dabei „zwischen alle nationalen Stühle“ - wie übrigens viele Autoren der Prager deutschen Literatur seiner Zeit.

Im Hinblick auf das Romantikthema halte ich noch ein anderes Motiv für wichtig und erwähnenswert, da es Sauer selbst in seiner fragwürdigen Körner-Rezension unaufhörlich umkreist: Die Körnersche - nicht genügend ehrerbietige - Darstellung der Klassiker. Körner war zwar sicher kein ,Klassikerschänder und -verhöhner' (wie etwa die expressionistischen Dichter), es „ergab sich“ ihm nur, gleichsam unter der Hand, aus seiner Lektüre der Werke romantischer Programmatiker heraussteigend, ein „anderes“ Klassikerbild, das Sauer, der damalige „Hüter des Goethe-Schatzes“ nicht gelten lassen wollte und durfte:

Aus allen Ecken und Enden wird Klatsch und Tratsch und Schmutz zusammengekehrt; auch vom Niedrigsten und Unbedeutendsten bleibt der Leser nicht verschont [...] Anmassung und Respektlosigkeit auf der einen, Dünkel auf der anderen Seite als die treibenden historischen Kräfte [...]. Jedes fachliche Urteil wird als Ergebnis von persönlicher Stimmung oder Laune, gekränktem Ehrgeiz, verletzter Eitelkeit usw. aufgefaßt. Die Ewigkeit ist verschwunden, der Augenblick regiert. Es gibt nur gegenseitigen Neid und Hass, Charakterlosigkeit und Tücke, Treulosigkeit und Hinterlist [...] Gesinnungslosigkeit ist die Gesinnung unserer größten Schriftsteller, Charakterlosigkeit der Charakter unserer Literatur der zweiten Bluetezeit. (Sauer 1925: 145f.)

Für Sauer, dem die Klassiker, Goethe und Schiller, Himmelsgestirne waren, war die Klassikerdarstellung Körners eine Heiligtumschändung, die eben mit einer - religiösem Eifer gleichkommenden - Brutalität bekämpft werden musste.

Ferner gehörte Körner zum „falschen Lager“, da er nämlich eine andere theoretisch-methodologische Linie vertrat als Sauer und zugleich Fürsprecher hatte, die Sauers Widersacher auf dem germanistischen Spielfeld waren. Körner, der Schüler Minors und Arnolds (und somit eher Nachfolger

15 Krolop (2004: 276) ärgert sich - völlig zu Recht -, dass diese seltene solidarische Geste der tschechischen Kollegen gegenüber einem deutschen Germanisten von Klausnitzer nicht erwähnt wurde. 
Scherers als Diltheys), ${ }^{16}$ hatte von Anfang an missmutig die Wende der Literaturgeschichtsschreibung zur geistesgeschichtlichen Linie verfolgt. An den Werken Ungers, Gundolfs, Sauers, Lamprechts, Nadlers, Bartels', Cysarz' u. a. störte ihn - liest man seine Rezensionen - nicht so sehr die Tatsache, dass sie die Literaturgeschichte mit der Geschichte des Geistes und somit mit der deutsch-nationalen Konzeption aufs engste verbinden, nicht so sehr, dass sie dadurch das Objekt der Literaturgeschichte ins Uferlose erweitern (es um alle national-kulturellen und national-geistigen Erscheinung und Kontexte anreichern), nicht so sehr, dass sie der Literaturgeschichtsschreibung eine neue (mit jeglicher Wissenschaft inkompatible) Funktion zuschreiben, nämlich die erzieherische und appelative Funktion, sondern vor allem, dass sie bei alledem das feste Fundament der philologischen Akribie verlassen, die sorgfältige philologische Arbeit am Text durch spektakuläre zwar, doch meist spekulative und philologisch unkontrollierbare Geisteskonstruktionen ersetzen.

Körners wissenschaftliches Credo hieß demgegenüber: auf detaillierte Arbeit mit Quellen und Texten zu bauen, sich nicht mit bekannten und bereits hundertmal interpretierten Texten zufrieden zu geben, sondern in Archiven und Sammlungen unermüdlich neue Quellen aufzudecken, nicht mit Fakten aus zweiter Hand zu hantieren, sondern sich von der Richtigkeit eigener Thesen immer durch den Blick in den Text zurück zu überzeugen, keine spektakulären theoretischen Luftschlösser zu bauen, bevor eine anständige historisch-kritische Bearbeitung existiert, die Wahrheit der Quellen und Texte nicht irgendwelchen Systemzwängen, übersichtlichen und gut ausschauenden Typologien oder gar Ideologien zu opfern usw. In Körners Studien und Rezensionen gibt es unzählige Stellen, welche diese Überzeugungen belegen - und gegen alle Widersacher verteidigen. Angesichts Körners kritischer Heftigkeit ist es kein Wunder, dass er sich mit seinem philologischen Credo viele Feinde schuf, die ihn als Positivisten, Detailisten, „Mikrologen“, Pedanten und Haarspalter beschimpften. Unter ihnen auch August Sauer, ${ }^{17}$ der aber durch Körner sowie

16 Zu Körners Einschätzung Scherers vgl. seinen Aufsatz Wilhelm Scherer 1841-1886 (1916) und seine Rez. zu Wolfgang Kaysers Das sprachliche Kunstwerk. (1949) (beides in Körner 2001: 42-54, 168-184). Körner teilt zwar die allgemeine kritische Einstellung zu Scherers Positivismus, lobt aber andererseits Scherer für seine Erudition und Präzision im Umgang mit Fakten und für seine Zielstrebigkeit, mit welcher er versuchte, die Germanistik auf ein festes und exaktes methodologisches Fundament zu stellen. Zugleich kritisiert er die Kluft, die die spätere Germanistik zwischen Scherer und Dilthey aufriss, betont im Gegensatz dazu die relative Nähe der beiden Konzeptionen und die tiefe gegenseitige Achtung beider Forscher.

17 Der aber - im Gegensatz zu so manchem seiner Schüler - noch ein anständiger Philologe war, was ihm Körner ja auch mehrmals zubilligt: Gemessen an der Wildheit der Angriffe 
mittels Körner zugleich seine akademischen Widersacher bekämpfte, hauptsächlich wohl Oskar Walzel und Hermann August Korff.

Diese genuin philologische Methode und der - häufig defensiv motivierte - Zwang, sich selbst, sein eigenes Werk innerhalb der Überfülle an Methoden und Konzepten exakt zu verorten, ließ Körner andererseits als zuverlässigen Editor und Kommentator hervorstechen und zum „Chronisten“ der wissenschaftlichen Germanistik der ersten Hälfte des 20. Jahrhunderts - in der Gestalt seines Bibliographischen Handbuchs des deutschen Schriftums ${ }^{18}$ - werden.

Neben der Rezeption theoretischer Ansätze anderer trachtete Körner jahrelang, seine eigene Interpretationsmethode zu erarbeiten und zu formulieren. Die endgültige Abrundung und Veröffentlichung scheiterte jedoch an den Zeitumständen:

Gerade in dem Augenblick, da ich bezügliche Vorarbeiten von nahezu zwei Jahrzehnten ein systematisches Buch zu runden mich anschickte, bereits mit einem bedeutenden Verlage abgeschlossen und baldiges Erscheinen der , anthropologischen' Poetik, ,Dichtung als Ausdrucksgebilde` angekündigt hatte, bereitete die Weltkatastrophe diesen stolzen Plänen ein Ende, politische Diskriminierung machte mich für ein volles Jahrzehnt bürgerlich wie literarisch tot. [...] Inzwischen sind mir andere mit manchem zuvorgekommen, z. B. Emil Staiger. (Körner 2001: 168-185, 175)

Einen Einblick in die Ausrichtung der Methode ermöglicht wohl Körners Aufsatz Erlebnis - Motiv - Stoff von 1924 (publiziert signifikanterweise im Jahre der Habilitationsaffäre in der Festschrift für Oskar Walzel). Hier weist Körner - in enger Anlehnung an Freuds Traum- und Neurosendeutungen - der Interpretationswissenschaft die Aufgabe zu, für jeden Dichter einen „Motivkatalog“ zu erstellen und von ihm rückblickend auf das konstituierende „affektive Erlebnis“ zu schließen, das das Kunstwerk entstehen ließ. Körner überwindet durch seine psychoanalytisch fundierten Formulierungen den positivistischen Biographismus seiner Lehrer, reiht sich in die Schar der Motivforscher ein, bleibt aber zugleich abseits der modernen strukturalistischlinguistischen Strömungen. Es ist allerdings schwer zu sagen, welche endgültige

gegen Nadler und Cysarz sind Körners Attacken gegen Sauer eher selten und mild. Einer der wenigen direkten Angriffe ist z. B. in Körners Rezension zu Hermann Groenewegs Schrift über Jakob Julius David zu lesen: „Ich fürchte, zu solchen Albernheiten und Abwegen ist der Verfasser nicht aus eigenem Ungeschick gekommen, sondern verführt durch eine Methode, die [...] letzten Endes auf Sauers Schule zurückführt.“ (Körner 1931: 32)

18 An diesem Handbuch arbeitete Körner (angeleitet durch Oskar Walzel) sein ganzes Leben lang, indem er seit den Kriegsjahren so gut wie jede germanistische Schrift las und dokumentierte. Das Handbuch, herausgegeben erst 1949, bis heute gut benutzbar, ist somit als einer der Höhepunkte des Körnerschen Werkes einzustufen. 
Gestalt die Theorie/Methodologie Körners Ende der 30er Jahre angenommen ${ }^{19}$ und ob sie die Nachkriegsgermanistik in irgendeiner Weise beeinflusst hätte.

\section{Das Mährische}

Die mystischen Mähren-Bezüge Körners wurden eingangs schon besprochen. Meine Feststellung, Körner habe „wenig Mährisches“ geschrieben, beziehe ich hier auf den Aufsatz zur deutschmährischen Literatur, nämlich auf Körners Besprechung der Schrift Hermann Groenewegs, J.J. David in seinem Verbältnis zur Heimat, Geschichte, Gesellschaft und Literatur aus dem Jahre 1931, die allerdings viel mehr ist als eine bloße Rezension, nämlich ein Bekenntnis „zum guten Lande Mähren“, zu dessen ,sanft-schwermütigen, widerstandsschwachen, sinnenfroh-nachdenklichen, [...] mehr betrachtsamen als tatkräftigen" (Körner 1931: 29) ${ }^{20}$ Bewohnern und zur Literatur, die dieses Land gebar. Da Körner die rezensierte Schrift Groenewegs für zwar „fleißig“, doch „albern“, „geistlos“, „oberflächlich“ und ohne die „Kenntnis von Land und Leuten“ geschrieben hielt, ersetzte er das Fehlende durch eine eigene Studie zur deutschmährischen Literatur, zum „deutschmährischen Triumvirat“, wobei er - spannenderweise - Jakob Julius David für den größten der Triumvieren hält:

Was hier [Davids Ruzena Capek; IFF] und in wenigen ähnlichen Stücken gleicher Art geboten ist, läßt ähnliche Arbeiten Saars und der Ebner weit hinter sich. (Körner 1931: 30)

David siegt bei Körner über die beiden reiferen, berühmteren, vollendeteren mährischen Dichter dank dreierlei Vorzügen:

Erstens durch das Sozial-Empfindsame und Volksnahe, das ihm weder der „distinguierte Wiener Saar, der das mährische Dorf und seine Bewohner, deren Sprache ihm unbekannt und unsympathisch war, nur von außen gesehen und als Kuriosität so betrachtet wie dargestellt," noch die Ebner, die „als hochgeborene Gräfin der Gesellschaftsstufe der Dörfler zu weit entrückt [war]" gleich machen konnten.

19 Ob Körners geplante Schrift Dichtung als Ausdrucksgebilde zumindest als Manuskript - etwa im Marbacher Archiv unter weiteren Nachlassstücken - vorliegt, weiss ich nicht.

20 Auch diese Betrachtung des „typisch mährischen“ Menschenschlags hat in der späteren Forschung Schule gemacht (Fiala-Fürst 2007). 
Zweitens durch „das starke Ethos“ und „den tieferen Ernst [...] des mit eigenem Herzblut genährten Schaffens“ (Körner 1931). Nach der Lektüre bereits weniger Schriften Körners fällt es auf, was für einen hohen Wert er allgemein und wiederholt der Authentizität und der ethischen Dimension literarischer Werke beimisst - und hier eben auch den Werken Davids zuerkennt.

Drittens durch die Nähe zu dem anderen Idiom und der anderen Kultur Mährens, dem Tschechischen: Nicht genug daran, dass Körner Davids tiefe Kenntnis der mährischen (auch der tschechischen) Realien und seinen schöpferischen Umgang mit der slawischen Mundart lobt, er erklärt ihn kurzerhand zum ,beinah“ tschechischen Dichter: Im Geburtsjahr Davids (1859) galt das Tschechische noch ,als Stapfe, als Stigma des niederen Volkes, des Vulgus. [...] Ein Menschenalter später und David wäre ein tschechisches Dichter geworden." Körner scheint es gar leid zu tun, dass David nicht - im Gegensatz zu Karel Hynek Mácha ${ }^{21}$ - den Sprung vom deutschen zum tschechischen Dichter geschafft hat:

Es stellt sich die Frage oft ein, ob dieses ganze Werk sich nicht noch besser, wurzelhafter, echter - und größer in tschechischer Sprache ausgenommen hätte. (Körner 1931: 31)

Angesichts solcher Äußerungen kommen mir Spekulationen ob der Körnerschen Haltung zum Tschechischen als doppelt müßig vor, da - zumindest auf der Ebene der kulturellen Beziehungen - längst beantwortet. In provokanter Art würde ich sogar die These aufstellen: Ein Menschenalter später, einen Krieg und eine Vertreibung weniger und Körner wäre ein tschechischer Germanist geworden. Schon seine - bereits erwähnten - häufigen, immer wohlwollenden Stellungnahmen zu den Arbeiten seiner Prager tschechischen Kollegen, auch die Tatsache, dass er wiederholt in tschechischen wissenschaftlichen Periodika und auf tschechisch publizierte, lassen Körner in einem ganz anderen Licht und Kontext erscheinen, als seine Prager deutschen Kollegen und Kontrahenten Sauer, Nadler und Cysarz.

Ein anderes Mähren-Kapitel wäre mit dem Vergleich der beiden aus Mähren stammenden germanistischen Zeitgenossen, Körner und Cysarz, aufzuschlagen - was allerdings Otokar Fischer bereits 1937 tat. In seinem Aufsatz Gelehrtentypen in der Prager Presse vergleicht Fischer das Werk der beiden Forscher und obzwar er Cysarz mit einigen positiven Adjektiven bedenkt, („die stürmischere Persönlichkeit“, „,der berühmtere Fachgenosse“, „,die anspruchsvollere Methode“), entdeckt er doch sofort mit spürendem Auge die Gefahr des Tendenziösen, welche aus Cysarz' Werk hervorquillt:

21 Máchas - von Otokar Fischer dargestellten - Weg vom Deutschen zum Tschechischen zieht Körner als Vergleichsbasis heran (Körner 1931: 31). 
[Cysarz] faßt die Wissenschaft nicht nur als Erforschung des Vergangenen, vielmehr handhabt er sie als Waffe im aktuellen Ringen um ein neu sich formendes Weltbild [...] im Ekstatisch-Methodologischen, im Rhetorischen [ist er] suggestiv, ja berückend und mit der Überredungskunst eines Rattenfängers von Hameln begabt. (Fischer 1937)

Fischers Sympathien liegen eindeutig bei der Methode, dem Werk und der Person Körners. Außerdem erlaubt sich Fischer noch eine kleine Bosheit gegenüber Cysarz: Er verbindet nämlich beide, Cysarz wie Körner, mit dem Tschechischen („tschechisiert“ sie gleichsam), indem er - mit sichtlichem Vergnügen - Josef Körner als ,aus der Geburtsstadt T.G. Masaryks“ stammend und Cysarz als „Nachkommen wohl nicht deutscher Vorfahren aus dem Ostrauer Bezirk“ (Fischer 1937) apostrophiert. Während Körner dieser Bezug wahrscheinlich nichts ausmachte, hat sich Cysarz sicher schwarz geärgert.

Körners eigene Stellungnahmen zu Cysarz gehören dann zu dem überhaupt Schärfsten, was Körner je geschrieben hat. In seiner Rezension auf Cysarz' Opus Deutsche Barockdichtung von 1924 liest man Sätze, die das Herz eines braven Germanisten in der heutigen politisch korrekten Zeit höher schlagen lassen:

Gänzlich in Verruf geraten ist die heilsame alte [...] Sitte, daß junge Kraft erst am harten Holz historisch-kritischer Detailforschung arbeiten lerne, ehe sie an souveräne Synthesen sich wage. Wie haben sich die Zeiten geändert! Was sonst Abschluss und Ernte eines reichen Forscherlebens war, stellt man heut gleich an den Beginn der wissenschaftlichen Laufbahn, zwanzigjährige Jünglinge deuten und erschließen uns schon in ihren Doktorarbeiten die ,Probleme und Lebensformen von Hamann bis Hegel' oder ,Das Wesen der deutschen Romantik'. [...] Wo man mit Analysen noch kaum begonnen hat, will er schon die Synthese erzwingen, als ob man ein Haus einzurichten begönne, ehe der Dachstuhl aufgesetzt worden [...]

Was aber Geduld und Nerven des Lesers auf die härteste Probe stellt, ist des Buches eigenwillige, übertriebene barocke Sprache, die aber danach angetan ist, den Gedanken zu verbergen, oder doch zu verdunkeln, als ihn zu offenbaren [...] Da wird das selber schon sehr laute Substantivum noch durch ein oder mehrere grelle Adjektive überschrien, daß man vor lauter Lärm schließlich kein Wort mehr versteht [...] Dieser ,brillante' Stil ermüdet schon nach wenigen Seiten, er blendet das geistige Auge in solchem Maße, daß man bei längerem Lesen nichts mehr wahrnimmt als ein undeutliches Geflimmer [...] Cysarz kommt es nicht darauf an, ob er den Nagel auf den Kopf trifft oder daneben haut; ihm ist der Lärm die Hauptsache $[\ldots]$

Dennoch hat man nach beendeter Lektüre das Gefühl, als hätten wieder einmal kreiBende Berge ein armsäliges Mäuslein geboren. Es wird ein ungeheures Thema gesetzt, aber es wird im geringsten nicht gelöst, es wird bestenfalls umschrieben [...] [es ist] nicht mehr reine und also minderwertige Wissenschaft. (Körner 2001: 106-116, 107ff.)

Angesichts solcher Sätze nimmt es wunder, dass Cysarz zwei, drei Jahre nach Erscheinen dieser Rezension (1926) Körner habilitieren ließ. Nun, er „ließ“ nicht, er hat wohl - dazu von der akademischen Öffentlickeit dazu gezwun- 
gen - müssen. Als späte Rache an dem damals bereits besiegten und verstorbenen Körner lassen sich dann Cysarz' sehr herablassende, gewollt unterkühlte, ironisch schmähende Äußerungen zu Körner in seinen Erinnerungen Zehn Jahre Prag lesen.

Im nächsten „Mähren-Kapitel“ wird dank Körner der große Wiener Arthur Schnitzler an Mähren gebunden: ${ }^{22}$ Körner verfolgte Schnitzlers Werk bereits als Student der Wiener Germanistik, persönliche Bekanntschaft mit Schnitzler machte er kurz nach dem 1. Weltkrieg. Er traf ihn und korrespondierte mit ihm regelmäßig - auch noch aus Prag - schrieb über ihn einige Studien und zeitgenau zu Schnitzlers 60. Geburtstag ein ganzes Buch (Körner 1921).

Während die Romantikforscher sich noch heute gerne zu Körner melden - wenn sie ihn denn kennen - wollen die heutigen Schnitzlerianer nichts mehr von ihm wissen: Einer anderen Forschergeneration angehörend, andere Theoreme und Methoden benutzend, v. a. mit erotischen Topoi anders (nach der sexuellen Revolution der 70er Jahre) umgehend und wahrscheinlich demselben „Persönlichkeitskult“ verfallen wie Sauer dem Goetheschen, „beleidigen sich“23 die heutigen Schnitzler-Forscher ob mancher negativer Einschätzungen Schnitzlers aus der Feder Körners und halten Körners Blick auf das Schnitzlersche Werk allgemein für unobjektiv, moralisierend, archaisch, verflossen - gar für antimodernistisch, völkisch und antisemitisch(! ${ }^{24}$

Seiner Interpretationsmethode treu sucht Körner in Schnitzlers Werk motivische Konstanten, die Einheit des Werkes, das tiefe Erlebnis, Ethos, Ernst und Authentizität - und findet sie häufig verdeckt durch Frivolität, unverbindliches Spiel, L'art pour l'artismus und gezwungene Skandalträchtigkeit, wobei er allerdings dem Spätwerk Schnitzlers die Hinwendung zu diesen - in seinen Augen - wichtigen Werten eines literarischen Kunstwerks zuerkennt (Körner 1927). Schnitzler selbst respektierte (überraschenderweise?) Körners Einschätzungen. Nach dem Erscheinen des Geburtstagsbuches notierte er in sein Tagebuch (1920-1922):

[ich las] mit grossem Interesse und wechselnden Gefuehlen... In den einzelnen Capiteln voll tief eindringenden Verständnisses, - mit seltnem Blick für die Zusammenhänge, die

22 Auch dem Schnitzler-Thema bei Körner widmet sich Ralf Klausnitzer sehr ausgiebig: Nur der Vollständigkeit halber (und für Leser, die diesen Aufsatz nicht parallel mit Klausnitzers [2001] Nachwort lesen werden) fasse ich nur seine Ausführungen zusammen - und bereichere sie um einen einzigen, hypothetischen, weiteren Mährenbezug.

23 Sprachschöpfung von Friedrich Torberg.

24 Diese und ähnliche unsinnige und ohne die Kenntnis des Körnerschen Gesamtwerkes getätigte Äußerungen aus dem Buch Konstanze Fliedls (1997) kritisiert Ralf Klausnitzer in seiner behutsamen und vornehmen Art. Dem ist nichts hinzuzusetzen. 
Einheit meines Schaffens - ich interessierte mich gewissermassen selbst und spürte ohne Eitelkeit das ,bleibende'. (zit. n. Klausnitzer 2001: 407)

Zum Zerwürfnis zwischen Schnitzler und seinem viel jüngeren Kritiker Körner kam es erst, als Körner Schnitzlers Werk mit dem von Jakob Julius David verglich (und hier ist eben der Mähren-Bezug) und es als „zu leicht befunden" hat. Eben beim Vergleich der Bearbeitung erotischer Motive von David und Schnitzler gibt Körner eindeutig David „bessere Noten“, bevorzugt den „überzüchteten Verführungsszenen“ Schnitzlers Davids „keusches Stillschweigen“, dem „Pessimismus der Blasiertheit“ Schnitzlers den „Pessimismus der Lebensschwere“ Davids und nennt - angesichts Davids , naturgetreuer Widergabe“ des mährischen Idioms - Schnitzlers „Salonsprache“ konventionell, „dünn und blaß“ (Körner 1931: 31f.). ${ }^{25}$

Das sind für die (großteils affirmative) Schnitzler-Forschung ungewohnt harte Worte und gemessen an der Entwicklung, die den einen Autor zum literarischen Himmel emporwachsen ließ und den anderen in fast vollkommene Vergessenheit stürzte, scheint es, dass Körner tatsächlich ,aufs falsche Pferd gesetzt" hat. Doch Körners, auf der Grundlage einer nahen, privaten Beziehung aufbauenden, eigenwillige Schnitzler-Interpretationen erinnern mich (und das ist der nächste und letzte Mähren-Bezug) an die ähnlich ungewohnte und eigenwillige Wittgenstein-Interpretation des Olmützers Paul Engelmann, der auch glaubte, Wittgensteins Tractatus besser als andere verstanden zu haben, weil er Wittgenstein als Freund näher stand als andere (Janik 1999; Václavek 1999).

25 Ich frage mich, wieso bisher noch keinem Literaturhistoriker eingefallen ist, dass Schnitzlers beleidigte Reaktion gerade ob dieser konkreten Studie (Körner hat weiß Gott Schlimmeres über Schnitzler geschrieben) vielleicht mehr mit David als mit Körner zusammenhängen könnte. David war - wie man von Hermann Bahr weiß - alles andere als beliebt unter den Wiener Modernisten. Den Beziehungen zwischen Schnitzler und David bin ich allerdings nicht nachgegangen. 


\section{Das Jüdische}

$\mathrm{Ob}$ und wie und inwiefern die jüdische Herkunft Körners der Grund für die Querelen mit Sauer war, wurde bereits besprochen. Einen weiteren erhellenden Blick auf Körners Beziehung zur jüdischen Problematik und auch zum eigenen Judentum bieten seine Beiträge zur deutsch-jüdischen Literatur, zum jüdischen Thema in der deutschen Literatur. In dieser Sparte hat Körner einiges geleistet, indem er beispielsweise - trotz seiner großen Zuneigung zur Romantik - die starke antisemitische Tendenz einiger romantischer Dichter redlich vermerkte und kritisierte: So wirft er im Nachwort zu seiner Herausgabe einer bisher unbekannten Erzählung Brentanos, Die Schachtel mit der Friedenspuppe, Brentano einen ,judenfeindlichen, antisemitischen Entrechat" vor (Brentano 1922: 67), fasst im Jahre 1930 - in einem leider viel zu kurzem Aufsatz - vollkommen richtig und auf sehr moderne Art die Gründe für die Entstehung des romantischen Antisemitismus und die antisemitischen Äußerungen der Romantiker um die Christlichdeutsche Tischgesellschaft zusammen, kreidet noch im Jahre 1942 (!) der deutschen Literaturgeschichtsschreibung an, dass „sie zu lange die Augen schloss vor den antisemitischen Ansichten und Handlungen der Mehrheit der Romantiker" (Körner 1942: 234) ${ }^{26}$ und die Beziehung der Romantik zur jüdischen Problematik nicht genügend untersuchte. Die „, brutale Gesinnung in der Judenfrage“ (Körner 1930: 123) seines Lieblingsromantikers Arnim straft er mit einer pointierten Schmähung Arnims, die auf den ersten Blick als schnell und kurz erzählte Anekdote anmutet, eigentlich aber über Jahre gewachsen ist: Während nämlich die „Antikriegshaltung“ Arnims, über die sich Körner und Kafka (vermutlich auf der Grundlage des Körnerschen Aufsatzes Achim von Arnim und der Krieg, 1915) im Jahre 1917 brieflich unterhalten haben (Härtl 2000), hier noch als positiver Charakterzug Arnims dargestellt wird:

So gibt er sich [Arnim; IFF] keineswegs romantischen Illusionen über das blutige Handwerk hin, berauscht sich nie auf Fouques alberne Weise in billiger Hurrahpoesie, sondern spricht rundweg von der, gemeinen Menschenschlächterei, die wir mit dem Namen Krieg benennen'. (Körner 1915: 340)

26 Abgesehen davon, dass bereits die Tatsache, dass „der Jude Körner“ noch 1942 unter seinem Namen publizieren durfte (obschon , nur' in einer tschechischen Zeitschrift), ein Rätsel ist, ist freilich ein weiteres Rätsel, wieso ein Aufsatz dieses - projüdischen - Inhaltes erscheinen konnte. 
Damit wird die pazifistische Zurückhaltung Arnims 15 Jahre später (im Aufsatz Romantischer Antisemitismus) in den Kontext der schandhaften Duellaffäre Arnims gegen den Juden Moritz Itzig gesetzt und zur Geschichte einer feigen und ordinären Kriegsverweigerung umpointiert:

Und wie sonderbar nimmt sich die Geschichte erst aus, wenn wir neben den gekränkten Juden, der im Jahre 1813, seine höchste Bürgerpflicht erfüllend, bei Lützen gefallen ist, den hochmütigen Beleidiger stellen, den der überlaut bekannte deutsch-christliche Patriotismus nicht gehindert hat, dem großen Volkskriege fernzubleiben. (Körner 1930: 123)

Lange Jahre hat sich Körner mit dem Plan herumgetragen, eine HeinrichHeine-Monographie zu schreiben, ${ }^{27}$ die allerdings - wie vieles andere Geplante - durch Nazitum, Krieg und Nachkriegszeit verhindert wurde. Ein tschechisch verfasster Aufsatz über Heine aus dem Jahre 1947 deutet vielleicht die Stoßrichtung, Zielsetzung und Atmosphäre der geplanten Monographie an: Zwei Haupteigenschaften Heines, zwei grundlegende Lebens- und Werkmotivationen werden hier hervorgehoben: Heines - durch scharfen Intellekt und unkäufliche Luzidität bedingte - lebenslange Weigerung, bei ideologischen Strömungen und Gruppierungen lange oder bedingungslos mitzutun ${ }^{28}$ und Heines nur scheinbarer Abfall vom Judentum, den Körner als eine seiner vielen Übeltaten [,darebáctví"] deutet, die einzige aber, die Heine - laut Körner - wirklich tief und lebenslang bereute. ${ }^{29}$

27 Vgl. Briefe an Käte Hamburger vom 15.3.1948 und 8.4.1948 (Körner 2001: 273). Spannend wäre der Frage nachzugehen, ob Körner Brods Heine-Monographie gekannt hat, die bereits im Exil-Verlag Allert de Lange in Amsterdam 1934 erschien und womöglich nicht so schnell auf den tschechischen Büchermarkt kam.

28 „Avšak bdělé vědomí a bystrý kritický rozum mu vždycky velmi záhy dávají prohlédnout každou lákavou iluzi [...] Tak se stává špatným soudruhem [...] opouští všechny tyto bojové tábory, k nimž patřil vždy jen napolo a jež se mu uprošred boje z duše ošklivily. “ [Doch sein waches Bewusstsein und sein kritischer Verstand ließen ihn immer sehr bald jede lockende Illusion durchschauen [...] So wird er zum schlechten Genossen [...], der all die Kampflager, zu denen er jeweils nur halbherzig gehörte und die er inmitten des Kampfes bereits verabscheute, verlässt.] (Körner 1947: 366)

29 „Takovým darebáctvím - které sám nikdy jinak neposuzoval - byl Heinův křest. Uvažujemeli z hlediska sociálně-dějinného o situaci, v níž se pochopitelně, ba nutně jeví nikoli největším darebáctvím jeho života, přesto však jediným, kterého opravdu lituje a přes něž se nikdy nepřenesl, na něž stále vzpomíná s uprímnou lítostí a s trapným studem, nikdy s urážlivým cynismem, který doprovází jiné hanebnosti jeho pozemské pouti.“ [Eine solche Übeltat - nie hat es Heine anders gedeutet - war seine Taufe. Aus der Sicht der sozial-historischen Situation erscheint die Taufe als verständliche Tat und nicht notwendig als die größte Übeltat seines Lebens. Doch für Heine ist es die einzige, die er wirklich bereute, sie nie verschmerzte, sie unaufhörlich mit Scham- und Reuegefühlen erinnerte, sie nie durch beleidigenden Zynismus verklärte - wie so oft die anderen Übeltaten seiner irdischen Laufbahn.] (Körner 1947: 366) 
Auf Körners Lebenssituation im Jahre 1947 blickend (von der die Briefe an Käte Hamburger beredtes Zeugnis geben), die Situation nach der Shoa, in der ,befreiten' Tschechoslowakei, die sich rigoros ihrer Deutschen (auch wenn's deutsche Juden waren) entledigte, kann man vielleicht vermuten, dass Körners Heine-Artikel viel mehr ist als ein Gelegenheitsaufsatz zum Geburtstag eines toten Dichters, nämlich - besonders in den Passagen über die missglückte Assimilation, das „,neue Marranentum“ nach der Haskala, den scharfen Schmerz und die tiefe Beleidigung ob der Zurückweisung der Juden von den Deutschen - eine subjektive und empfindsame Äußerung zur eigenen jüdischen Identität und Seinsweise.

\section{Abschluss - keine Zusammenfassung: Romantik zum vierten}

Die haarsträubend falsche, weil logisch, rationell gesehen unmögliche Interpretation von Friedrich Hölderlins berühmtestem Gedicht, Hälfte des Lebens, ist zugleich - zumindest unter Studenten - die verbreitetste und bestechendste, weil sie irrationale, mystische, unabmessbare Tiefen auftut, indem sie nämlich suggeriert, Hölderlin ahnte in seiner Dichter-Genialität, dass er die zweite Hälfte seines Lebens, „wenn es Winter ist“, im Turm verbringen würde. Auch Josef Körner schrieb ein ,Hälfte-des-Lebens-Gedicht', das zwar bei weitem nicht die Mehrbödigkeit und Großartigkeit des Hölderlinschen Gedichts hat und Körners Leben auch nicht so spektakulär halbiert wie das Hölderlins, das aber immerhin Ende 1931 geschrieben wurde, also knappe eineinhalb Jahre bevor Hitler in Deutschland an die Macht kam und einen menschenvernichtenden Mechanismus in Bewegung brachte, der in Körners Leben und Schaffen einen Bruch verursachte, welcher nicht mehr zu überbrücken war. 
Dieses Gedicht bilde - unkommentiert - den Abschluss dieses Beitrages:

Wehen

War dies alles nur Beginn

Oder geht es schon zu Ende?

Stehe vor der großen Wende

Zum hinan ich, zum dahin?

Bricht nach Leid und fehlem Trachten,

Nach Verdüstrung und Verzicht

In mein Dasein nun das Licht

Oder will es vollends nachten?

Wer den Sinn der Stunde wüßte!

Ringt aus schwer gewordnem Schoß

Sich ein neues Leben los?

Geht ein altes früh zu Rüste? (Körner 1931)

\section{Literatur}

Boden, Petra (1994): Julius Petersen. Ein Wissenschaftsmanager auf dem Philologenthron. - In: Euphorion 88, 82-102.

Brentano, Clemens (1922): Die Schachtel mit der Friedenspuppe. Hrsg. v. Josef Körner. Wien, Prag, Leipzig: Strache.

Brod, Max (1918): Prager Dichterschule? - In: Der Friede 1918/19, 14.

Brod, Max (1966): Der Prager Kreis. Stuttgart, Berlin, Köln, Mainz: Kohlhammer.

Cysarz, Herbert (1957): Zehn Jahre Prag. - In: Jahn, Rudolf (Hg.), Gren ₹fall der Wissenschaft: Herbert Cysarz: Frankfurt/M.: Heimreiter.

Fiala-Fürst, Ingeborg (2007): Das Thema der jüdischen Assimilation. - In: Slawinski, Ilona (Hg.), Der Mnemosyne Träume. Festschrift zum 80. Geburtstag Joseph P. Strelkas. Tübingen: Francke.

Fiala-Fürst, Ingeborg/Krappmann, Jörg, Léblová, Silvie (Hgg.) (2002): Lexikon deutschmäbrischer Autoren. Beiträge zur deutschmäbrischen Literatur. Bd. 5. Olomouc: Univ.-Verl. Univerzita Palackého.

Fischer, Otokar (1937): Gelehrtentypen. - In: Prager Presse (3.1.1937, Sonntagsbeilage). 
Fliedl, Konstanze (1997): Arthur Schnitz̨ler. Poetik der Erinnerung. Wien, Köln, Weimar: Böhlau.

Fohrmann, Jürgen (1989): Das Projekt der deutschen Literaturgeschichte. Stuttgart: Metzler.

Härtl, Heinz (2000): Zu Kafkas Briefen an Josef Körner über Arnim. Mit Körners Artikel ,Achim v. Arnim und der Krieg` als Anhang. - In: Ehlers, Klaas-Hinrich/ Höhne, Steffen/Nekula, Marek/Maidl, Václav (Hgg.), Brücken nach Prag. Festschrift für Kurt Krolop zum 70. Geburtstag. Frankfurt/Main: Lang, 321-346.

Horkheimer, Max (1938): [ohne Titel]. - In: Zeitschrift für Sozialforschung 7, 220.

Janik, Allan (1999): Die Rolle Engelmanns in Wittgensteins philosophischer Entwicklung. - In: Schneider, Ursula (Hg.), Paul Engelmann. Architektur, Judentum, Wiener Moderne. Wien, Bozen: Folio, 39-56.

Klausnitzer, Ralf (2001): Nachwort. - In: Körner, Josef, Philologische Schriften und Briefe. Hrsg. von dems. Göttingen: Wallstein, 385-461.

Körner, Josef (1915): Achim von Arnim und der Krieg. - In: Der Zeitgeist, Beiblatt zum Berliner Tageblatt [Nachdruck in Härtl (2000: 338-342)].

Körner, Josef (1917a): Franz Werfel. - In: Die Tat 9, 776 f.

Körner, Josef (1917b): Rez. zu Georg Kaiser: Die Versuchung. - In: Donauland 1, 762.

Körner, Josef (1917c): Dichter und Dichtung aus dem deutschen Prag. - In: Donauland 1 [Nachdruck in Körner (2001: 55-66)].

Körner, Josef (1918): Rez. zu Paul Adler: Die Zauberflöte. - In: Donauland 2, 359.

Körner, Josef (1919): Metahistorik des deutschen Schrifttums. - In: Deutsche Rundschau [Nachdruck in Körner (2001: 84-87)].

Körner, Josef (1921): Arthur Schnitzllers Gestalten und Probleme. Zürich, Leipzig, Wien: Amalthea.

Körner, Josef (1923): Der Narr der Liebe. - In: Preußische Jabrbücher 191, 52.

Körner, Josef (1924): Romantiker und Klassiker. Die Brüder Schlegel in ihren Beziehungen zu Schiller und Goethe. Berlin: Askan. Verl. [Reprint: WBG Darmstadt 1971].

Körner, Josef (1924): Erlebniß - Motiv - Stoff. - In: Wahle, Julius/Klemperer, Viktor (Hgg.), Vom Geiste neuer Literaturforschung. Festschrift für Oskar Walzel. WildparkPotsdam: Athenaion, 80-90.

Körner, Josef (1927): Artur Schnitzlers Spätwerk. - In: Preußische Jahrbücher 208, 53$83,153-163$.

Körner, Josef (1930): Romantischer Antisemitismus. - In: Jüdischer Almanach auf das Jahr 5691 [Nachdruck in Körner (2001: 122-126)].

Körner, Josef (1931): Rez. zu Hermann Groeneweg: J.J. David in seinem Verhältnis zur Heimat, Geschichte, Gesellschaft und Literatur. - In: Literaturblatt für germanische und romanische Philologie 52, 29-37. 
Körner, Josef (1931b): Wehen. - In: Deutsche Zeitung Bohemia (29.11.1931).

Körner, Josef (1942): Nové prameny pro poznání německé romantiky [Neue Quellen zum Verständnis der deutschen Romantik]. - In: Casopis pro moderní filologii 28, 230-236.

Körner, Josef (1947): Věrný apostata. Ke 150. narozeninám Heinricha Heineho [Ein treuer Apostat. Zum 150. Geburtstag Heinrich Heines]. - In: Vèstnik židovské obce náboženské v Praze 9/18, 365-366.

Körner, Josef (1949): Bibliographisches Handbuch des deutschen Schriftums. Bern: Francke.

Körner, Josef (2001): Philologische Schriften und Briefe. Hrsg. v. Ralf Klausnitzer mit einem Vorw. v. Hans Eichner. Göttingen: Wallstein. ${ }^{30}$

Krolop, Kurt (2004): Ein Pionierprojekt, aber keine Pionierleistung. - In: brücken NF $12,265-290$.

Sauer, August (1925): Rezension zu Josef Körner: Romantiker und Klassiker - In: Euphorion 26, 142-150.

Sokel, Walter H. (1969): Expressionismus als Literatur. Bern, München: Francke.

Václavek, Ludvík (1999): Der Engelmann-Kreis in Olmütz. - In: Schneider, Ursula (Hg.), Paul Engelmann. Architektur, Judentum, Wiener Moderne. Wien, Bozen: Folio, 37-52.

30 Klausnitzers Band (Körner 2001) beinhaltet folgende Aufsätze Körners: Die Renaissance des germanischen Altertums, 11-41; Wilhelm Scherer 1841-1886, 42-54; Dichter und Dichtung aus dem deutschen Prag, 55-66; Arthur Schnitzlers Gestalten und Probleme, 67-83; Metabistorik des deutschen Schrifttums, 84-87; Die Wiener „Friedensblätter" 1814-1815, eine romantische Zeitschrift, 88-105; Barocke Barockforschung, 106-116; Auferstehende Romantik! Bericht über die neu entdeckten Romantiker-Briefe, 117-121; Romantischer Antisemitismus, 122-126; Acbim von Arnim und Frau von Staël, 127-132; Persönliche Erinnerungen an Arthur Schnitzler, 133-136; Ideologie und Humanität, 137162; Rezension Otto Mann: Der Junge Friedrich Schlegel, 163-167; Rezension Wolfgang Kayser: Das sprachliche Kunstwerk, 168-184. 
